\section{Author's response: co-trimoxazole treatment in idiopathic pulmonary fibrosis}

We thank Neto et $\mathrm{l}^{1}$ for their comments on our paper ${ }^{2}$ and for reiterating the points contained within it. 'The survival benefit conferred by co-trimoxazole, if real, could be due to its antimicrobial activity as there was a significant reduction in the number of infections in the group receiving active treatment', and this is likely to occur more frequently in patients receiving immunosuppressive treatment. The proportion of deaths in the intention-to-treat group on immunosuppression was 29 of 37. As stated, 'this study was not designed to collect microbiological information' however, the results were adjusted for baseline azathioprine or mycophenylate use, and a subgroup analysis of deaths by immunosuppression treatment at baseline in the perprotocol population was undertaken which could not detect a subgroup effect. Analyses from the intention-to-treat and per-protocol (PP) populations were prespecified, and reporting was appropriate.

As discussed, the results could be 'due to increased mortality in those withdrawing from the drug because of side effects, or the higher withdrawal rate in the active group could be a marker of the disease severity. However, the reduction in mortality was not due to a disproportionate withdrawal of patients in the treatment arm immediately prior to death, as only four patients (two from each group) withdrew from the study within 1 month of death.' It would be inappropriate to present the results of the demographic details from the PP population, given the desire not to put emphasis on this type of analysis.

Likewise, the issue regarding patients' diagnosis has been discussed. The study commenced prior to current diagnostic criteria, however, a sensitivity analysis was undertaken of patients with probable or definite usual interstitial pneumonia (UIP) defined as 'honeycombing on the high resolution CT scan, a histopathological diagnosis of UIP or predicted to have a histopathological diagnosis of UIP' according to the criteria of Fell et al, ${ }^{3}$ the results of which were similar to the full analysis.

There is a paucity of clinical trials evaluating prophylactic antibiotics in patients with interstitial lung disease, although Enomoto et $a l^{4}$ showed a benefit with co-trimoxazole prophylaxis against Pneumocystis jiroveci pneumonia in patients with interstitial pneumonia receiving high-dose glucocorticoid therapy. From our study, the odds ratio of having an infection, corrected for azathioprine usage, was 2.17 for patients with more than $10 \mathrm{mg} /$ day prednisolone, compared with those not receiving prednisolone in the control group, and 2.14 in the intervention group. The rate ratio was similar to that found by Greenberg et $a l^{5}$ (1.30) with reduced rates of infection in the intervention group $(70 \%$ vs $42 \%)$. Interestingly, there were high rates of infection in the control group of patients with idiopathic interstitial pneumonia even in those not receiving prednisolone (62\%).

As stated clearly in the manuscript, the study did not detect a beneficial effect in terms of the primary endpoint. However, we believe that the magnitude of effect on survival in the PP analyses means that treatment with co-trimoxazole in patients with idiopathic pulmonary fibrosis warrants further investigation.

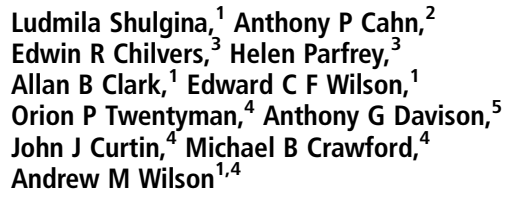

${ }^{1}$ Norwich Medical School, University of East Anglia, Norwich, UK 
${ }^{2}$ Department of Respiratory Medicine, Bedford Hospital NHS Trust, Bedford, UK

${ }^{3}$ Respiratory Medicine Division, Department of Medicine, School of Clinical Medicine, University of Cambridge, Adenbrooke's/CUHNHSFT and Papworth Hospitals, Cambridge, UK

${ }^{4}$ Department of Respiratory Medicine, Norfolk and Norwich University Hospital NHS Foundation Trust, Norwich, UK

${ }^{5}$ Department of Respiratory Medicine, Southend University Hospital NHS Foundation Trust, Essex, UK

Correspondence to Dr Andrew Wilson, Department of Medicine, Norwich Medical School, University of East Anglia, Norwich NR4 7TJ, UK; a.m.wilson@uea.ac.uk

Competing interests None.

Provenance and peer review Commissioned; internally peer reviewed.
To cite Shulgina L, Cahn AP, Chilvers ER, et al. Thorax 2013;68:884-885.

Received 19 April 2013

Accepted 25 April 2013

Published Online First 21 June 2013

\section{SLinked}

- http://dx.doi.org/10.1136/thoraxjnl-2013-203395

Thorax 2013;68:884-885.

doi:10.1136/thoraxinl-2013-203765

\section{REFERENCES}

1 Ribeiro Neto ML, Swigris JJ, Culver DAl. Idiopathic pulmonary fibrosis or not: antibiotic prophylaxis for all patients on immunosuppressants. Thorax 2013;68:884-5.

2 Shulgina L, Cahn AP, Chilvers ER, et al. Treating idiopathic pulmonary fibrosis with the addition of co-trimoxazole: a randomised controlled trial and economic evaluation. Thorax 2013;68: 155-62.

3 Fell CD, Martinez FJ, Liu LX, et al. Clinical predictors of a diagnosis of idiopathic pulmonary fibrosis. Am J Respir Crit Care Med 2010;181:832-7.

4 Enomoto T, Azuma A, Matsumoto A, et al. Preventive effect of sulfamethoxasole-trimethoprim on Pneumocystis jiroveci pneumonia in patients with interstitial pneumonia. Intern Med 2008:47:15-20.

5 Greenberg JD, Reed, Kremer JM, et al. Association of methotrexate and tumour necrosis factor antagonists with risk of infectious outcomes including opportunistic infections in the CORRONA registry. Ann Rheum Dis 2010;69:380-6. 